\title{
A DUALITY THEOREM FOR A MULTIPLE OBJECTIVE FRACTIONAL OPTIMIZATION PROBLEM
}

\author{
T. WEIR
}

\begin{abstract}
Characterizations of efficiency and proper efficiency are given for classes of multiple objective fractional optimization problems. These results are then applied to the case of multiple objective fractional linear problems. A dual problem is given for the multiple objective fractional problem and it is shown that for a properly efficient primal solution the dual solution is also properly efficient.
\end{abstract}

\section{Introduction}

In a recent paper [9] the author proposed a dual for a convex multiple objective optimization problem relating properly efficient solutions of the primal and the dual. Duality for nonconvex problems was also considered. However, for such nonconvex problems, it was pointed out, that the dual solution corresponding to a properly efficient primal solution could not be guaranteed to be properly efficient.

In this paper characterizations of efficiency and proper efficiency are given for classes of multiple objective fractional problems. Such problems are generally not convex. The results specialize to a known result for multiple objective linear fractional problems: for linear fractional problems, all efficient solutions are properly efficient. For the nonlinear multiple objective fractional problem, a dual is proposed, in which the objective function is the same as that of the primal multiple objective fractional problem. For this dual problem, it is

Received 13 January, 1986.

Copyright Clearance Centre, Inc. Serial-fee code: 0004-9727/86 $\$ A 2.00+0.00$. 
shown that the dual solutions are properly efficient.

\section{Efficiency and proper efficiency}

Consider the multiple objective optimization problem

$$
\text { 'maximize' } F(x) \text { subject to } h(x) \geqq 0 \text {. }
$$

where $X$ is an open convex subset of $\mathrm{R}^{n}$ and where $F: X \rightarrow \mathrm{R}^{k}$ and $h: X \rightarrow R^{m}$ are real vector valued functions. This is the problem of finding the set of efficient or Pareto [7] optimal points for (P): $x_{0}$ is said to be efficient if it is feasible for (P) and there exists no other feasible point $x$ for such that $F(x) \geqslant F\left(x_{0}\right)$ and $F(x) \neq F\left(x_{0}\right)$.

The notion of proper efficiency introduced by Kuhn and Tucker [3] is a restriction of efficiency that allows only points $x_{0}$ such that there is no solution to

$$
\begin{cases}\nabla F_{i}\left(x_{0}\right) d>0 & \text { for some } i ; \\ \nabla F_{j}\left(x_{0}\right) d \geqslant 0 & \text { for all } j \neq i ; \\ \nabla h_{j}\left(x_{0}\right) d \geqslant 0 & \text { for all } j \in J\end{cases}
$$

where $J=\left\{i: h_{i}\left(x_{0}\right)=0\right\}$ and where the Kuhn-Tucker constraint qualification [3] is satisfied. Their motivation was that of excluding solutions admitting a first order gain in one criterion at the expense of but a second order gain in another.

Geoffrion's concept of proper efficiency [2] is a slightly restricted definition of efficiency which eliminates the points causing unbounded tradeoffs between the objectives $\left\{F_{i}\right\}: x_{0}$ is to be properly efficient if it is efficient for (P) and if there exists a scalar $M>0$ such that, if $F_{i}(x)>F_{i}\left(x_{0}\right)$ for some $i$ and feasible $x$ for $(P)$, there exists $j$ such that $F_{j}(x)<F_{j}\left(x_{0}\right)$ and

$$
\frac{F_{i}(x)-F_{i}\left(x_{0}\right)}{E_{j}\left(x_{0}\right)-F_{j}(x)} \leqslant M .
$$

In this section we provide characterizations of efficiency for several classes of multiple objective optimization problems. Results for linear fractional problems will be given as special cases. In the 
following all functions will be assumed differentiable. We denote by $E$ the set of efficient points and by $P E(K T)$ and $P E(G)$ the set of efficient points that are properly efficient in the sense of Kuhn and Tucker and Geoffrion respectively.

LEMMA 1. If $x_{0}$ is efficient for $(P)$ and if $F$ and each $h_{j}, j \in J$, are pseudoconvex at $x_{0}$ then there is no solution to the system (1).

Proof. Suppose there exists $d$ satisfying (1). Then for $t \in(0,1)$, $\nabla F_{i}\left(x_{0}\right)\left(x_{0}+t d-x_{0}\right)>0 ;$ The pseudoconvexity of $F_{i}$ at $x_{0}$ then implies

$$
F_{i}\left(x_{0}+t d\right)>F_{i}\left(x_{0}\right) .
$$

Similarly for all $j \neq i,(1)$ and the pseudoconvexity of $F_{j}$ at $x_{0}$ implies that for $t \in[0,1]$

$$
F_{j}\left(x_{0}+t d\right) \geqslant E_{j}\left(x_{0}\right) \text {. }
$$

Similarly, for all $j \in J$ and for $t \in[0,1]$

$$
h_{j}\left(x_{0}+t d\right) \geqslant h_{j}\left(x_{0}\right)=0 \text {. }
$$

For $j \notin J, h_{j}\left(x_{0}\right)>0$ and by continuity there is $\bar{t}$ where $0<\bar{t}<1$ such that for $t \in\left[0, Z_{]}\right.$

$$
h_{j}\left(x_{0}+t d\right)>0 .
$$

Combining the results (2) - (5) contradicts the efficiency of $x_{0} \cdot 0$

It is to be noted that the assumption in Lemma 1 on $h_{j}, j \in J$, is the weak reverse convex constraint qualification. This constraint qualification implies the kuhn-Tucker constraint qualification [4].

The next result may be found in Geoffrion [2].

LEMMA 2. There is no solution to (1) if and only if there exists $\bar{\lambda} \in \mathrm{R}^{k}$ and $\bar{y} \in \mathrm{R}^{m}$ such that

$$
\nabla \sum_{i=1}^{k} \bar{\lambda}_{i} F_{i}\left(x_{0}\right)+\nabla \sum_{i=1}^{m} \bar{y}_{i} h_{i}\left(x_{0}\right)=0
$$




$$
\begin{gathered}
\sum_{i=1}^{m} \bar{y}_{i} h_{i}\left(x_{0}\right)=0 \\
\bar{\lambda}>0, \sum_{i=1}^{k} \bar{\lambda}_{i}=1, \bar{y} \geqq 0 .
\end{gathered}
$$

Combining Lemmas 1 and 2 gives

LEMMA 3. If $x_{0}$ is efficient for $(P)$ and if $F$ and each $h_{j}$, $j \in J$, are pseudoconvex at $x_{0}$ then there exists $\bar{\lambda} \in R^{k}$ and $\bar{y} \in R^{m}$ such that (6), (7) and (8) are satisfied.

Assuming $F$ and $h$ concave and that the Kuhn-Tucker constraint qualification is satisfied Geoffrion showed that (6), (7) and (8) are satisfied at $x_{0}$ if and only if $x_{0} \in P E(G)$. Under the same hypotheses Kuhn and Tucker showed that (6), (7) and (8) are satisfied if and only if $x_{0} \in P E(K T)$. Thus for a concave problem (P), if the Kuhn-Tucker constraint qualification is satisfied, $P E(G)=P E(K T)$. The next results extend the results of Geoffrion and Kuhn and Tucker to $a$ class of multiple objective fractional optimization problems.

Consider the problem

$$
\text { 'maximize' } F(x) \quad \text { subject to } \quad h(x) \geqq 0 \text {. }
$$
Here $E_{i}(x)=\frac{f_{i}(x)}{g_{i}(x)}, i=1,2, \ldots, k$ where each $f_{i}: X \rightarrow R$ and $g_{i}: X \rightarrow \mathrm{R}, i=1,2, \ldots, k$ are concave and convex functions respectively with $f_{i}(x) \geqslant 0, g_{i}(x)>0$ for all $x \in X$; each $g_{i}$, $i=1,2, \ldots, k$ is assumed to be bounded on $X$ and each $h_{i}, i=1,2, \ldots, n$ is concave. Note that each $E_{i}, i=1,2, \ldots, k$ is pseudoconcave but, in general, not concave on $X$.

THEOREM 4. For the problem (FP), if the Kuhn-Tucker constraint qualification holds at $x_{0}$, there exists $(\bar{\lambda}, \bar{y})$ such that $(6),(7)$ and (8) are satisfied if and only if $x_{0} \in P E(G)$.

Proof. $\Leftrightarrow$ Let $x$ be any feasible point for (FP). 
Then from (7) and (8) $\sum_{i=1}^{m} \bar{y}_{i} h_{i}(x)-\sum_{i=1}^{m} \bar{y}_{i} h_{i}\left(x_{0}\right) \geqslant 0$ and concavity of $\sum_{i=1}^{m} \bar{y}_{i} h_{i}(\cdot)$ implies that $\nabla \sum_{i=1}^{m} \bar{y}_{i} h_{i}\left(x_{0}\right)\left(x-x_{0}\right) \geqslant 0$. Thus, from (6), $\sum_{i=1}^{k} \frac{\bar{\lambda}_{i}}{g_{i}\left(x_{0}\right)}\left(\nabla f_{i}\left(x_{0}\right)-\frac{f_{i}\left(x_{0}\right)}{g_{i}\left(x_{0}\right)} \nabla g_{i}\left(x_{0}\right)\right)\left(x-x_{0}\right) \leqslant 0$.

write $\phi_{i}(x)=\frac{\bar{\lambda}_{i}}{g_{i}\left(x_{0}\right)}\left(f_{i}(x)-\frac{f_{i}\left(x_{0}\right)}{g_{i}\left(x_{0}\right)} g_{i}(x)\right), i=1,2, \ldots, k$; the concavity assumptions imply that each $\phi_{i}, i=1,2, \ldots, k$ is concave and so $\sum_{i=1}^{k} \phi_{i}$ is concave.

Thus $\sum_{i=1}^{k} \frac{\bar{\lambda}_{i}}{g_{i}\left(x_{0}\right)}\left(f_{i}(x)-\frac{f_{i}\left(x_{0}\right)}{g_{i}\left(x_{0}\right)} g_{i}(x)\right) \leqslant 0$. Consequently $x=x_{0}$ is a global maximizer for the problem

$$
\underset{x \in X}{\operatorname{maximize}} \sum_{i=1}^{k} \frac{\bar{\lambda}_{i}}{g_{i}{ }^{\left(x_{0}\right)}}\left(f_{i}(x)-\frac{f_{i}\left(x_{0}\right)}{g_{i}\left(x_{0}\right)} g_{i}(x)\right)
$$

subject to $h(x) \geqq 0$.

The Comprehensive Theorem of Geoffrion [2] then shows that $x=x_{0}$ is an element of $P E(G)$ for the problem$$
\text { (P') 'maximize' } K(x) \text { subject to } h(x) \geqslant 0
$$

where $k_{i}(x)=f_{i}(x)-\frac{f_{i}\left(x_{0}\right)}{g_{i}\left(x_{0}\right)} g_{i}(x), i=1,2, \ldots, k$.

If $x_{0}$ was not an element of $P E(G)$ for $(F P)$ then for every $M>0$ there is a feasible point $x$ and an $i$ such that $\frac{f_{i}(x)}{g_{i}(x)}>\frac{f_{i}\left(x_{0}\right)}{g_{i}\left(x_{0}\right)}$ and

$$
\left[\frac{f_{i}(x)}{g_{i}(x)}-\frac{f_{i}\left(x_{o}\right)}{g_{i}\left(x_{o}\right)}\right] /\left[\frac{f_{j}\left(x_{o}\right)}{g_{j}\left(x_{o}\right)}-\frac{f_{j}(x)}{g_{j}(x)}\right]>M
$$

for all $j$ such that $\frac{f_{j}(x)}{g_{j}(x)}<\frac{f_{j}\left(x_{0}\right)}{g_{j}\left(x_{0}\right)}$. Hence 


$$
\left[f_{i}(x)-\frac{f_{i}\left(x_{0}\right)}{g_{i}\left(x_{0}\right)} g_{i}(x)\right] /\left[g_{j}(x) \frac{f_{j}\left(x_{0}\right)}{g_{j}\left(x_{0}\right)}-f_{j}(x)\right]>\left[\frac{g_{i}(x)}{g_{j}(x)}\right] \cdot M
$$

contradicting $x_{\mathrm{o}} \in P E(G)$ for $\left(\mathrm{P}^{\prime}\right)$.

$\Leftrightarrow$ Follows from the Comprehensive Theorem in [2]. 0

THEOREM 5. For the problem (FP), if the Kuhn-Tucker constraint qualification holds at $x_{0}$, there exists $(\bar{\lambda}, \bar{y})$ such that $(6),(7)$ and (8) are satisfied if and only if $x_{0} \in P E(K T)$.

Proof. $\Leftrightarrow$ The proof of Theorem 4 shows that if $(6),(7)$ and (8) are satisfied then $x_{0} \in P E(G)$; thus from [2], assuming the Kuhn-Tucker constraint qualification, $x_{0} \in P E(K T)$.

$\Leftrightarrow$ This is given in ([3], Theorem 4).

口

COROLLARY 6. Under the assumptions of either Theorems 4 or 5 $P E(G)=P E(K T)$ for the problem (EP).

Consider now the multiple objective linear fxactional problem (LFP)

$$
\text { 'maximize' } F(x) \text { subject to } A x \leqq b
$$

where $F_{i}(x)=\left(c_{i}^{t} x+\alpha_{i}\right) /\left(d_{i}^{t} x+\beta_{i}\right), c_{i} \in \mathrm{R}^{n}, d_{i} \in \mathrm{R}^{n}, \alpha_{i} \in \mathrm{R}$, $B_{i} \in \mathrm{R}$ and $d_{i}^{t} x+\beta_{i}>0$ for all $x \in X, i=1,2, \ldots, k$.

Since each $F_{i}$ is pseudoconvex on $X$, Lemma 3 and Theorem 4 imply

THEOREM 7. $x_{0}$ is an efficient solution for (LFP) if and only if $x_{0} \in P E(G)$ for $(L F P)$.

Similarly we have

THEOREM 8. $x_{0}$ is an efficient solution for (LFP) if and only if $x_{\circ} \in P E(K T)$ for $(L F P)$.

The result in Theorem 7 was first given by Choo [1]. However, there, only proper efficiency as defined by Geoffrion was considered.

COROLLARY 9. For problem (LFP), $E=P E(G)=P E(K T)$. 口 


\section{Duality}

The scalar valued fractional programming problem may be expressed as

$$
\underset{x \in X}{\operatorname{maximize}} \frac{f(x)}{g(x)} \quad \text { subject to } \quad h(x) \geqq 0 \text {. }
$$

Here $X$ is an open convex subset in $R^{n} ; f: X \rightarrow R$ and $g: X \rightarrow R$ are differentiable concave and convex functions respectively, with $f(x) \geqslant 0$, $g(x)>0$ for all $x \in X . h: X \rightarrow \mathrm{R}^{m}$ is a differentiable concave function. Various different dual problems to (F) have appeared in the literature - see for example Schaible [8] where many references are given. Following Mond and weir [6], a dual probram for (F) is:

$$
\text { minimize } \begin{aligned}
\frac{f(x)}{g(x)} \quad \text { subject to } & {\left[\frac{f(x)}{g(x)}\right]+\nabla y^{t} h(x)=0 } \\
y & \geqslant 0, y^{t} h(x) \leqslant 0 .
\end{aligned}
$$

In this section we define a concept of duality for the multiple objective problem and establish a duality theorem for (FP) giving multiple objective analogs of the duality results for (F) and (D). Here proper efficiency will be taken to be that defined by Geoffrion [2].

Given a problem

$$
\left(P^{\circ}\right) \quad \text { 'maximize' } \psi(x) \text { subject to } x \in S
$$

where $\psi: \mathrm{R}^{n} \rightarrow \mathrm{R}^{k}$ and $S \subseteq \mathrm{R}^{n}$, the problem

$\left(D^{\circ}\right) \quad$ 'minimize' $\phi(x)$ subject to $x \in T$

where $\phi: R^{\ell} \rightarrow R^{k}$ and $T \subseteq R^{\ell}$ will be called a dual for ( $P^{\circ}$ ) if

(i) (Weak Duality). Whenever $x$ is feasible for $\left(P^{\circ}\right)$ and $u$ feasible for $\left(D^{\circ}\right)$

$$
\psi(x) \neq \phi(u) .
$$

(ii) (Strong Duality). If $\left(P^{\circ}\right)$ has a properly efficient point $x_{0}$ then $\left(D^{\circ}\right)$ has a properly efficient point $u_{0}$ and $\psi\left(x_{0}\right)=\phi\left(u_{0}\right)$.

The above concept of duality was defined in [9] and there an analog of Wolfe's [10] duality theorem for convex multiple objective problems was given. However, it was pointed out, that, for nonconvex problems, a 
dual solution corresponding to a properly efficient primal solution could not be guaranteed to be properly efficient. For a dual problem to be defined for (FP) below it will be shown that the dual solutions are properly efficient.

In relation to (FP) consider the problem

(FD)

$$
\begin{aligned}
& \operatorname{'minimize'}_{x \in X} F(x) \quad \text { subject to } \nabla\left\{\sum_{i=1}^{k} \lambda_{i} \frac{f_{i}(x)}{g_{i}(x)}\right\}+\nabla y^{t} h(x)=0 \\
& y^{t} h(x) \leqslant 0 \\
& y \geqq 0, \lambda>0, \sum_{i=1}^{k} \lambda_{i}=0
\end{aligned}
$$

THEOREM 10 (Weak Duality). Let $x$ be feasible for $(F P)$ and $(u, y, \lambda)$ feasible for $(E D)$. Then $F(x) \neq F(u)$.

Proof. Suppose to the contrary that there is an $x$ feasible for (FP) and $(u, \lambda, y)$ feasible for (FD) such that $F(x) \geqslant F(u)$. Then for some $i \in\{1,2, \ldots, k\}$

$$
\frac{f_{i}(x)}{g_{i}(x)}>\frac{f_{i}(u)}{g_{i}(u)}
$$

and for all $j \neq i$

$$
\frac{f_{j}(x)}{g_{j}(x)} \geq \frac{f_{j}(u)}{g_{j}(u)}
$$

From the pseudoconcavity of each $F_{i}, i=1,2, \ldots, k$ it follows that

$$
\nabla\left[\sum_{i=1}^{k} \lambda_{i} \frac{f_{i}(u)}{g_{i}(u)}\right](x-u)>0 .
$$

Hence, using the equality constraint of (FD),

$$
\nabla y^{t} h(u)(x-u)<0 \text {. }
$$

But primal and dual feasibility gives

$$
\begin{aligned}
y^{t} h(x) & \geqslant 0 \text { and } y^{t} h(u) \leqslant 0 ; \text { thus, since } \mathrm{h} \text { is concave, } \\
& \nabla y^{t} h(u)(x-u) \geqslant 0 \text { - a contradiction. }
\end{aligned}
$$


THEOREM 11 (Strong Duality). Let $x_{0}$ be a properly efficient point (FP), and let the Kuhn-Tucker constraint qualification be satisfied.

Then there exists $(\bar{\lambda}, \bar{y})$ such that $\left(x_{0}, \bar{\lambda}, \bar{y}\right)$ is a properly efficient point for (FD) and the objectives of (FP) and (FD) are equal.

Proof. Since $x_{0}$ is properly efficient for (FP) and the Kuhn-Tucker constraint qualification is satisfied, Theorem 4 gives the existence of $\bar{\lambda}$ and $\bar{y}$ such that $\bar{\lambda}>0, \sum_{i=1}^{k} \bar{\lambda}_{i}=1, \bar{y} \geqq 0$ and

$$
\begin{gathered}
\nabla\left[\sum_{i=1}^{k} \bar{\lambda}_{i} \frac{f_{i}\left(x_{0}\right)}{g_{i}\left(x_{0}\right)}\right]+\nabla \bar{y}^{t} h\left(x_{0}\right)=0 \\
\bar{y}^{t} h\left(x_{0}\right)=0 .
\end{gathered}
$$

Thus $\left(x_{0}, \bar{\lambda}, \bar{y}\right)$ is feasible for (FD) and the objectives are equal. If $\left(x_{0}, \bar{\lambda}, \bar{y}\right)$ was not efficient for (FD) there would exist feasible $\left(u^{*}, \lambda^{*}, y^{*}\right)$ for (FD) such that, for some $i \in\{1,2, \ldots, k\}$

$$
\frac{f_{i}\left(u^{*}\right)}{g_{i}\left(u^{*}\right)}<\frac{f_{i}\left(x_{0}\right)}{g_{i}\left(x_{0}\right)}
$$

and for all $j \neq i$

$$
\frac{f_{j}\left(u^{*}\right)}{g_{j}\left(u^{*}\right)} \leqslant \frac{f_{j}\left(x_{0}\right)}{g_{j}\left(x_{o}\right)}
$$

Then, since each $E_{i}, i=1,2, \ldots, k$ is pseudoconcave

$$
\nabla\left[\frac{f_{i}\left(u^{*}\right)}{g_{i}\left(u^{*}\right)}\right]\left(x_{0}-u^{*}\right)>0
$$

and

$$
\nabla\left[\frac{f_{i}\left(u^{*}\right)}{g_{i}\left(u^{*}\right)}\right] \quad\left(x_{0}-u^{*}\right) \geqslant 0 .
$$

Hence, since $\lambda_{i}^{*}>0$ for all $i$,

$$
\nabla\left[\sum_{i=1}^{k} \lambda_{i} * \frac{f_{i}\left(u^{*}\right)}{g_{i}\left(u^{*}\right)}\right]\left(x_{0}-u^{*}\right)>0 .
$$

Using the equality constraint of (FD) then implies that $y^{*} h\left(u^{*}\right)\left(x_{0}-u^{*}\right)<0$. But primal and dual feasibility implies 
$y^{* t} h\left(x_{0}\right) \geqslant 0$ and $y^{* t} h\left(u^{*}\right) \leqslant 0 ;$ since $h$. is concave it follows that $\nabla y^{*} h\left(u^{*}\right)\left(x_{0}-u^{*}\right) \geqslant 0$ - a contradiction. Thus $\left(x_{0}, \bar{\lambda}, \bar{y}\right)$ is efficient for (FD). It remains to show that $\left(x_{0}, \bar{\lambda}, \bar{y}\right)$ is properly efficient. The proof of Theorem 4 and (9) and (10) shows that $x_{0}$ solves the problem

$$
\underset{x \in X}{\operatorname{maximize}} \sum_{i=1}^{k} \bar{\lambda}_{i}\left[\frac{f_{i}(x)}{g_{i}\left(x_{0}\right)}-\left[\frac{f_{i}\left(x_{0}\right)}{g_{i}\left(x_{0}\right)}\right] \frac{g_{i}(x)}{g_{i}\left(x_{0}\right)}\right]
$$

subject to $h(x) \geqq 0$.

The duality results of Mond and Weir [5] then shows that $\left(x_{0}, \bar{y}\right)$ is a solution for the problem

$$
\underset{x \in X}{\operatorname{minimize}} \sum_{i=1}^{k} \bar{\lambda}_{i}\left[\frac{f_{i}(x)}{g_{i}{ }^{\left(x_{0}\right)}}-\left[\frac{f_{i}\left(x_{0}\right)}{g_{i}\left(x_{0}\right)}\right] \frac{g_{i}(x)}{g_{i}\left(x_{0}\right)}\right]
$$

subject to

$$
\begin{aligned}
\nabla\left[\sum_{i=1}^{k} \bar{\lambda}_{i} \frac{f_{i}(x)}{g_{i}(x)}\right]+\nabla y^{t} h(x) & =0 \\
y^{t} h(x) & \leqslant 0 \\
y & \geqq 0 .
\end{aligned}
$$

As in the proof of Theorem 4, it then follows that $\left(x_{0}, \bar{\lambda}, \bar{y}\right)$ is properly efficient for (FD).

REMARK 12. It is to be noted that if $k=1$ then (FD) becomes the problem (D); thus (FD) is a multiple objective analog of (D).

\section{References}

[1] E.U. Choo, "Proper Efficiency and the Linear Fractional Vector Maximization Problem", Oper. Res., 32 (1984), 216-220.

[2] A.M. Geoffrion, "Proper efficiency and the theory of vector maximization", J. Math. Anal. Appl., 22 (1968), 618-630.

[3] H.W. Kuhn and A.W. Tucker, "Nonlinear Programming", Proceedings Second Berkeley Symposium on Mäthematical Statistics and Probability, (Univ. of California Press, Berkeley, California, 1950) 481-492. 
[4] O.L. Mangasarian, Nonlinear Programing, (McGraw-Hill, New York, 1969).

[5] B. Mond and T. Weir, "Generalized concavity and duality", 263-279 in:

S. Schaible and W.T. Ziemba (eds), Generalized Concavity in Optimization and Economics, (Academic Press, New York, 1981).

[6] B. Mond and T. Weir, "Duality for fractional programming with generalized convexity conditions", J. Inform. Optim. Sci., 3 (1982), 105-124.

[1] v. Pareto, Cours d'economie Politique (Rouge, Lausanne, 1896).

[8] S. Schaible, "Fractional programming. I. Duality", Management Sci., 22 (1976), 858-867.

[9] T. Weir, "Proper efficiency and duality for vector valued optimization problems", Department of Mathematics, Royal Military College, Duntroon, Research Report No. 12/85. (submitted for publication)

[10] P. Wolfe, "A duality theorem for nonlinear programming", Quart. Appl. Math., 19 (1961), 239-244.

Department of Mathematics, University College, Australian Defence Force Academy, Campbell, A.C.T. 2600. 